\title{
Research on Path Planning and Obstacle Avoidance for Tunnel Mobile Robot Based on Visual Navigation
}

\author{
Xiaolong Zhou, Xin Wan, Xiaoxue Guo \\ State Grid Chongqing Electric Power CO. Electric Power Research Institute, Chongqing 404100 \\ Xinping Li \\ The Smartech Institute, 518057, Shenzhen, China
}

\begin{abstract}
With the development of science and technology, the mobile robot has been widely concerned at home and abroad. Great potential for mobile robot applications. Applications include automatic driving, unknown field exploration, industry and agriculture production automation, dangerous environment no work, the mobile robot control technology development will have a profound impact on human life and production. Mobile robot is an intelligent system which integrates environmental information collection, image processing and object recognition, dynamic decision and planning, motion control and so on. Mobile robot through its own sensors to the external environment of information perception, acceptance and processing, to develop a walking route, and in the process of completing the process to avoid obstacles and other acts, and ultimately complete the task. In this paper, some key problems in the path planning and obstacle avoidance for tunnel robot are studied, such as visual distance measurement, path planning and obstacle avoidance.
\end{abstract}

KEYWORD: image processing; dynamic decision making and planning; tunnel robot

\section{INTRODUCTION}

Tunnel robot through the sensor sensing the external environment and its own state, to achieve in the obstacle environment, toward the goal of autonomous mobile complete certain work function of the robot system. With the development of science and technology, mobile robots become the focus of research at home and abroad, in the military, civil and scientific research in the various application widely, including aerospace, marine, military, service life of. Mobile robot is a collection of environmental information collection, image processing and object recognition, dynamic programming, motion control and other functions in one of the intelligent system. Machine vision, visual ranging, stereo matching, map reconstruction, path planning, target tracking, control strategies and machine control, etc.. In order to solve the monitoring work of the high dangerous environment of the tunnel, this paper studies the related functions of the tunnel robot.

\section{PATH PLANNING AND WALL BARRIER SYSTEM ARCHITECTURE FOR TUNNEL ROBOT}

According to the system function, the mobile robot system is mainly divided into two modules: video capture module, visual distance measurement module, road analysis and object extraction, and path planning and wall barrier module. Video capture module completed information collection, wireless transmission, sampling, frame synchronization function and the video format conversion, and enhance the system's performance, and binocular visual acquisition to the information and an infrared sensor to collect the data fusion. According to the principle of fuzzy control, the development of membership function and wall detection, the implementation of wall obstacle avoidance, and finally will plan a good angle, speed and other parameters to the PLC module to send a signal to control the robot's travel. Its schematic diagram is as follows: 


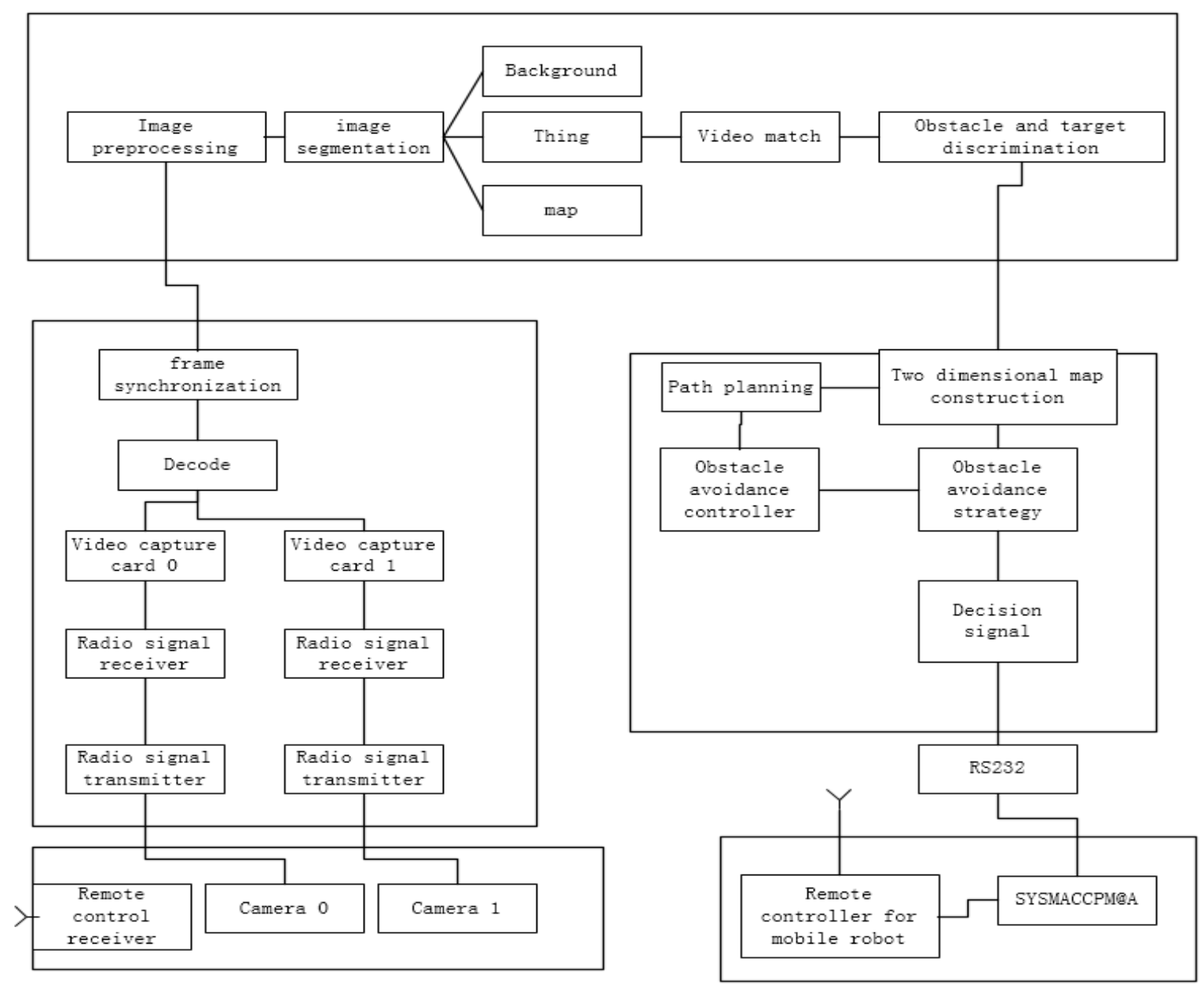

Fig.1 Vehicle control system

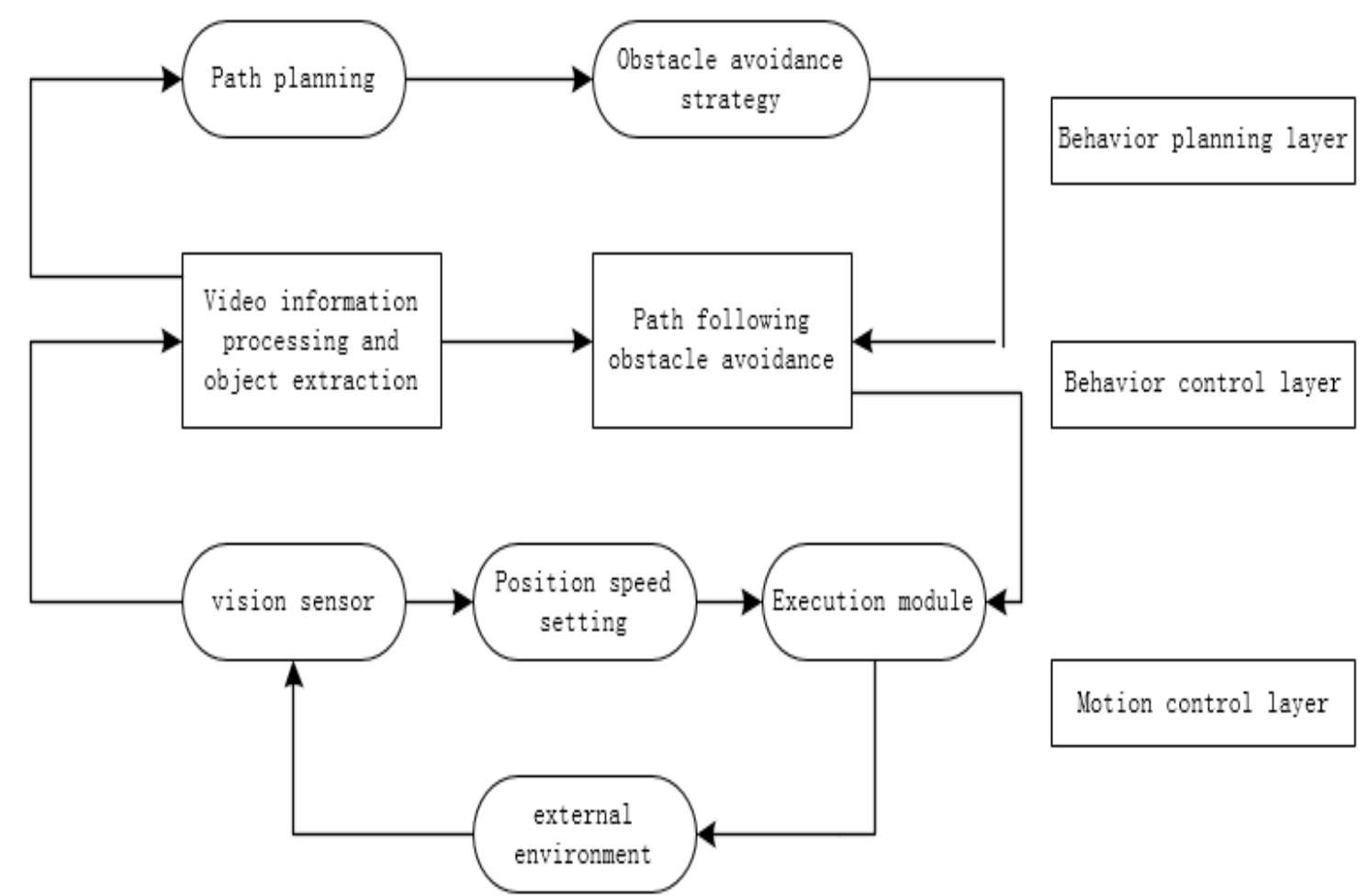

Fig.2 Structure of obstacle avoidance system 


\subsection{Control Section}

Robot control module consists of 1 OMRON SYSMAC CPM2A programmable controller and 1 sets of remote control transmitter, he will move the robot to carry out the signal conversion and PC and machine information exchange. Robot module is the main body of motion. The camera on the body to carry out the collection of environmental information, and through the wireless signal transmitter to send a video signal to the PC machine, PC machine to receive a series of information processing, and ultimately get a planned path. Will the results sent to exercise control signal to the machine control module and control module receives serial signal from the host module, cyclic monitoring in different registers of numerical, determine the control signal, and triggers the corresponding transport robot motion corresponds to end, emission corresponding control signal, the proper operation of the robot motion control. The system can be divided into three layers according to the hierarchy, which are: motion control layer, behavior control layer, behavior planning layer, as shown in figure 2.

In motion control layer, through the placement of the binocular camera on the robot body and placed in four outside the wheels of the infrared sensor to detect the environmental information, the fusion of information through the wireless signal transceiver to behavior control layer, data, analysis of environmental information, behavior control layer, including the information of road surface, obstacle avoidance and target depth information, size, shape and other parameters to be passed to the planning layer by a path planning module generated a path from the initial point to the target point, according to the obstacle avoidance strategy of obstacle avoidance behavior. During the course of the process, the control layer is guided by the corresponding coordinate value, the speed and the rotation angle. The physical appearance and structure of the robot are as follows:

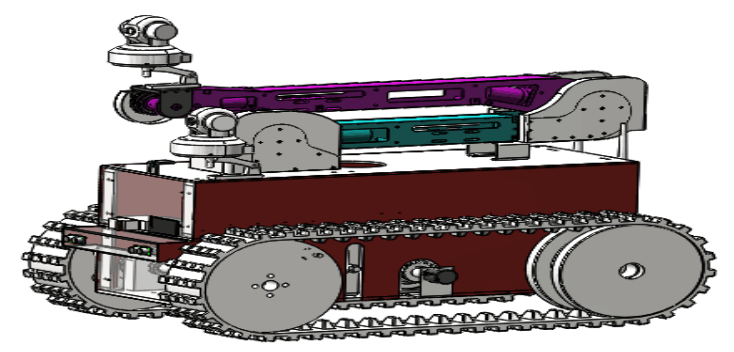

Fig.3 Structure diagram of tunnel robot

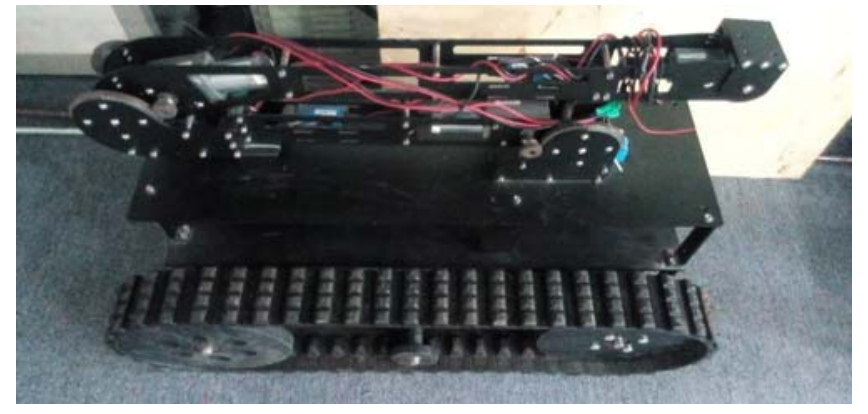

Fig .4 Real map of the tunnel robot

\section{PLC CONTROL SECTION}

PLC controller to run a cycle of monitoring procedures, monitoring the status register PLC. When the value of the specified register is in conformity with the trigger condition, the corresponding output end is communicated, and the remote signal transmitter is switched on, and the corresponding control signal is sent to the mobile robot. As shown in the figure, before and after the PLC output terminal respectively with the remote control signal transmitter is connected to the output signal of the left and right. PLC ground COM terminal and remote control signal transmitter connected to the ground wire. By sending RS232 serial signal, PC program control the output of PLC, so as to achieve the purpose of controlling the movement of the mobile robot.

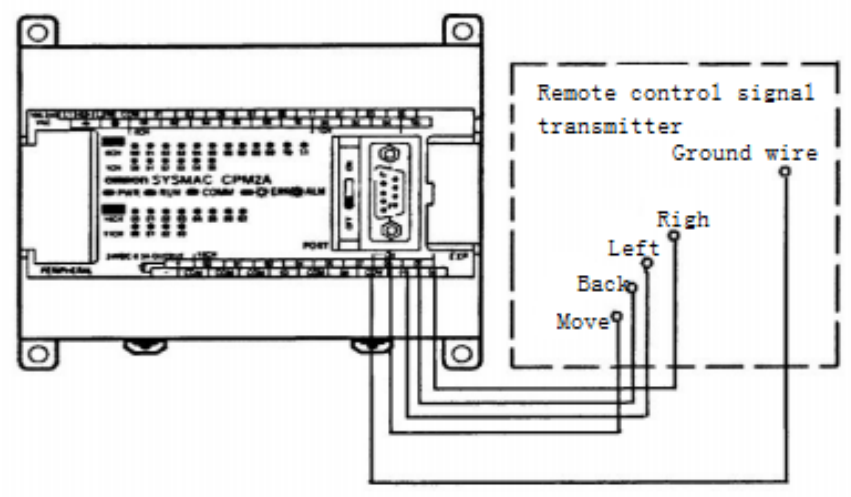

Fig.5 Schematic diagram of PLC control

\section{OBSTACLE AND OBJECT MAPPING EXPERIMENT}

Through the processing of image processing and object extraction module, the obstacle information is stored in a list of obstacles information which can be dynamically expanded by object pointer. A node represents an obstacle, including depth information of obstacles, the largest connected area, coordinate position, the location and size of the obstacles in the outer rectangle, and color information in the road 
segmentation module. Through this list, we will realize the construction of two-dimensional map.

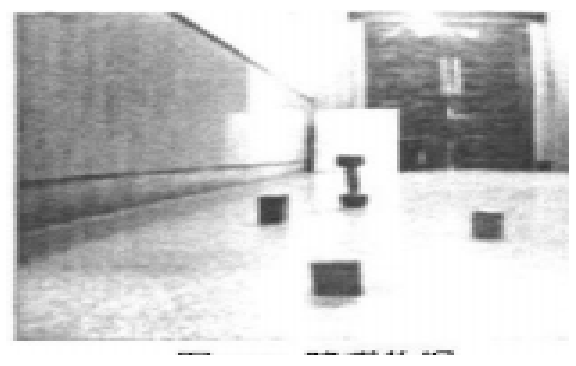

Fig.6 Obstacle

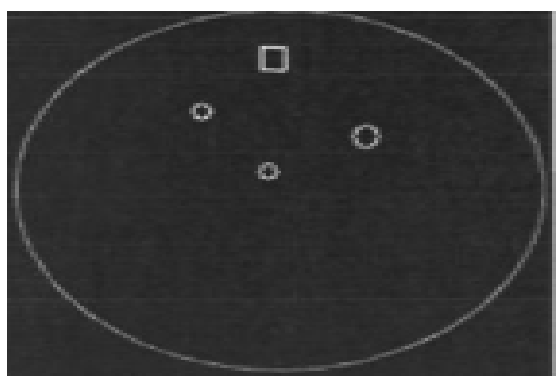

Fig.7 Obstacle map

After getting the position of the obstacle, the system realizes the calibration of the target position. The calibration of the target position is similar to that of the obstacle representation, and the position of the target is obtained according to the principle of similar triangle. The difference is that the goal of the calibration of the vector from the origin to the target location, to provide parameters for path planning. As the following figure is the vision of the state of the map.

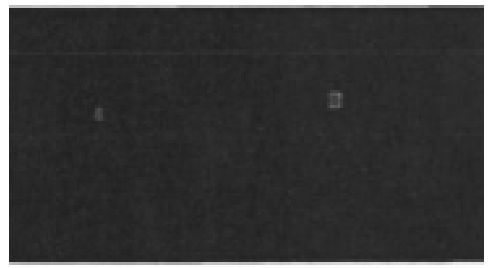

Fig.8 Long-range target

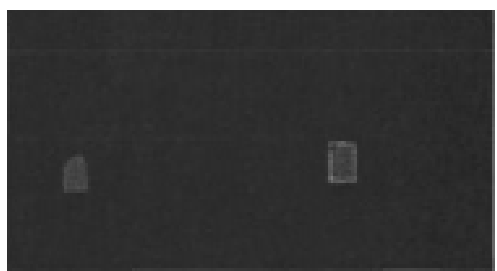

Fig.9 Close range object

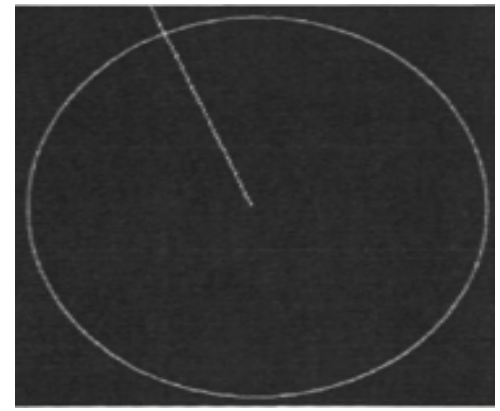

Fig.10Remote state map

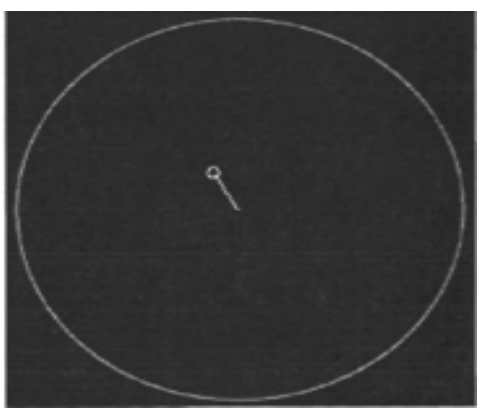

Fig.11Map of near distance

\section{CONCLUSION}

Tunnel robot path planning and obstacle avoidance system involving visual acquisition, multi-sensor data fusion, camera calibration, vision measurement, image processing, stereo matching, map building, obstacle and difference, route planning, avoid multiple avoidance strategies and machine control technology field. According to the actual needs of the project, precious several key problems, such as visual odometry, obstacles and target identification, path planning and obstacle avoidance strategy etc., indepth study, the tunnel robot using visual and infrared acquisition of environmental information, ranging, obstacles and map building, through the path planning and obstacle strategy to complete the demand for mobile robot remote control system and successfully avoid obstacles.

\section{REFERENCES}

[1] R Andrew Russell. Survey of Robotic Applications for Odorsensing Technology. The International Journal of Robotics Re-search, 2001, 20(2):144-162.

[2] Tsai RY. An efficient and accurate camera calibration technique for 3D machine vision[C].Proe.CVPR86.364-374. 